http://dx.doi.org/10.18778/2196-8403.2015.16

SUSANNE KAUL

\title{
Kafkas China
}

Kafka zajmuje się na początku XX wieku chińskimi zasadami funkcjonowania społecznego i chińskim ,sposobem myślenia‘. Jest to czas, w którym Chiny - ze względu na przemiany polityczne w kraju - faktycznie wzbudzają zainteresowanie na polu międzynarodowym. Jednak obraz Chin w twórczości Kafki jest ukształtowany przede wszystkim przez własne wyobrażenia autora. Artykuł ukazuje to na trzech przykładach: wierszu Jan-Tsen-Tsais Gtęboka noc, opowiadaniu Kafki Budowa chińskiego muru i paraboli Dschuang Dsis Sen o motylu.

Obwohl Kafka sich mit den gesellschaftlichen Gefügen und Denkweisen Chinas zu Beginn des 20. Jahrhunderts auseinandersetzt, in einer Zeit also, in der China aufgrund seiner politischen Umbruchsituation internationale Aufmerksamkeit auf sich zieht, ist sein Bild von China wesentlich durch sein eigenes dichterisches Selbstbild geprägt. Dies wird an drei Beispielen aufgezeigt: Jan-Tsen-Tsais Gedicht In tiefer Nacht, Kafkas Erzählung Beim Bau der chinesischen Mauer und Dschuang Dsis Gleichnis Der Schmetterlingstraum.

Although Kafka deals with China's social structures and ways of thinking at the beginning of the 20th century, that is, at a time when China's political upheaval gets international attention, his image of China is essentially characterized by its own poetic selfimage. This is to be shown on the basis of three examples: Jan-Tsen-Tsai’s poem „In the Depths of Night“", Kafka’s short story The Great Wall of China, and Dschuang Dsi’s parable Dreaming of a Butterfly.

\section{Einleitung}

Kafkas Bild von China ist weniger von einem politischen Interesse am Untergang des Kaisertums geprägt als vielmehr von ganz bestimmten Merkmalen, in denen er seine eigenen Denkfiguren und dichterischen Schaffensweisen wiedererkennt. Diese Strukturähnlichkeiten und Projektionen, die Kafkas Werk mit seinem Bild von China verbindet, werden hier in drei Unterkapiteln 
aufgezeigt, die Folgendes beinhalten: erstens Kafkas Positionierung zu JanTsen-Tsais Gedicht In tiefer Nacht, zweitens Kafkas Erzählung Beim Bau der chinesischen Mauer und drittens Dschuang Dsis Gleichnis Der Schmetterlingstraum, das exemplarisch mit Kafkas Text Die Wahrheit über Sancho Pansa verglichen wird. Die taoistische Einsamkeit des Schreibens, die unendlichen Hierarchien sowie die Paradoxien, die geläufige Vorstellungen auf den Kopf stellen, sind die wesentlichen Elemente, die Kafkas poetologisches Selbstbild und zugleich sein Bild von China prägen.

Der argentinische Schriftsteller und Kafka-Kenner Jorge Luis Borges nennt in einem Essay den chinesischen Schriftsteller Han Yu einen Vorläufer Kafkas. Zur Begründung dieser These führt er eine Fabel über ein Einhorn an und stellt fest, dass eine Verwandtschaft zu Kafka im Tonfall bestehe. Diesen wiederum kennzeichnet er als mysteriös und gelassen (BORGES 1992:118). ${ }^{1}$ Auch Kafka kreiert rätselhafte Mythen in aller stilistischer Ruhe, Lakonie und Prägnanz. Außerdem macht er häufig Gebrauch von Tieren in gleichnishafter Weise. Darauf weist auch Elias Canetti hin und deutet die Möglichkeit an, dass Kafkas Interesse für die chinesische Literatur, in denen Tiere häufig eine Rolle spielen, von Martin Bubers Buch Chinesische Geister- und Liebesgeschichten ${ }^{2}$ geweckt wurde. Auch CANETTI (1983:76) ist es wichtig zu sagen, dass Kafka nicht nur motivisch auf die chinesische Literatur zurückgreift: „Doch der einzige, seinem Wesen nach chinesische Dichter, den der Westen aufzuweisen hat, ist Kafka.“ Ob sich dieses Wesenhafte auf seine Person oder sein Schreiben bezieht, bleibt offen und kann offenbleiben, weil bei Kafka Schreiben und Person eins sind. Und gerade in dieser Identifikation sieht Kafka sich als Chinesen.

\section{Jan-Tsen-Tsai und die Nachtarbeit}

Kafka bezeichnet sich selbst als einen Chinesen. Auf einer Postkarte schreibt er im Mai 1916 an seine Geliebte Felice Bauer: „Ich denke, wenn ich ein Chinese wäre und gleich nach Hause fahren würde (im Grunde bin ich ja Chinese

1 Der Text Han Yus beschreibt in sachlichem Stil den distanzierten Umgang des Volkes mit der Vorstellung eines Einhorns, ohne die Bedeutung dieser Beschreibung zu explizieren.

2 Kafka kannte es, wie aus einer brieflichen Bemerkung gegenüber Felice Bauer im Januar 1913 hervorgeht, nur aus einer ausführlichen Rezension mit Textbeispielen. Vgl. WEIYAN MENG (1986:54). 
und fahre nachhause), müßte ich es doch bald erzwingen, wieder herzukommen.“ (KAFKA 2003:657)

Hintergrund dieser Bemerkung ist eine Reise nach Marienbad, die er später mit Felice gemeinsam wiederholen wird. Kafkas Identifikation mit einem Chinesen steht im Kontext seiner Beziehung zu Felice, die durch den Kampf zwischen der Einsamkeit des Schreibens und dem Eintritt in ein bürgerliches Eheleben gekennzeichnet ist. Diese Postkarte entsteht zwischen der ersten und der zweiten Verlobung bzw. Entlobung Kafkas mit Felice. Was bedeutet hier also die Bemerkung „im Grunde bin ich ja Chinese“? Das China-Motiv zieht sich durch die Korrespondenz mit Felice, seit Kafka auf ein Gedicht von Jan-TsenTsai, einem chinesischen Dichter des 18. Jahrhunderts, gestoßen ist. Das Gedicht lautet:

In tiefer Nacht

In der kalten Nacht habe ich über meinem

Buch die Stunde des Zubettgehens vergessen.

Die Parfüms meiner goldgestickten Bettdecke

sind schon verflogen, der Kamin brennt nicht mehr.

Meine schöne Freundin, die mit Mühe bis dahin

ihren Zorn beherrschte, reißt mir die Lampe weg

Und fragt mich: Weißt Du, wie spät es ist?

Das Gedicht hat Kafka in dem 1905 von Hans Heilmann herausgegebenen Band Chinesische Lyrik vom 12. Jahrhundert v. Chr. bis zur Gegenwart gefunden. Max Brod zufolge hat er oft begeistert aus dem Buch vorgelesen (vgl. KAFKA 2003:119). Kafka schreibt dieses Gedicht für Felice ab. In dem Brief, der das Gedicht enthält, verlangt er von ihr, die Nachtarbeit ihm zu überlassen. Sie solle nachts schlafen, nicht schreiben. Nur dann könne er nachts in Ruhe schreiben. Er brauche den Stolz auf die Nachtarbeit (vgl. KAFKA 2003:118). An dieser Stelle kommt das China-Motiv ins Spiel:

Aber warte einen Augenblick, zum Beweise dessen, daß die Nachtarbeit überall, auch in China den Männern gehört, werde ich aus dem Bücherkasten (er ist im Nebenzimmer) ein Buch holen und ein kleines chinesisches Gedicht für Dich abschreiben.

Er erklärt Felice, „daß die wohlhabenden Chinesen vor dem Schlafengehen ihr Lager mit aromatischen Essenzen parfümieren“, kommentiert das Gedicht an dieser Stelle sonst aber nicht weiter. Es wird nun deutlich, warum Kafka sich auf der Postkarte als Chinesen bezeichnet. Er identifiziert sich mit dem Gelehrten, der die Nacht Geschriebenem widmet und der folglich dem Schreiben und Lesen Priorität gegenüber dem Schlafen und Lieben einräumt. Kafka sieht seine Identität als Schriftsteller durch die Beziehung zu Felice bedroht und rät 
ihr wiederholt davon ab, ihn zu heiraten. Andererseits will er auf sie nicht verzichten. Das chinesische Gedicht dient hier also als Rechtfertigung einer Existenz, wie er sie sich wünscht: die Nacht der Dichtung widmen, ohne auf die Geliebte zu verzichten, die alles erduldet.

Daraus lässt sich jedoch kein China-Bild ableiten, denn Kafka erscheint das Männerbild des Nachtarbeiters universal. Gleichwohl manifestiert es sich in dem Chinesen dieses Gedichts, und so ist er eben dieser Chinese. Kafkas Sichtweise dieses Chinesen wird in späteren Briefen ausdifferenziert. Im Januar 1913 widmet er einen ganzen Brief der Deutung des Gedichts und weist darauf hin, dass von einer Freundin, nicht von einer Ehefrau die Rede sei. Im Falle einer Ehefrau wäre diese Nacht repräsentativ für das ganze gemeinsame Zusammenleben, „das ein Kampf um eine Lampe wäre“ (KAFKA 2003:262). Kafka verdeutlicht Felice damit den Kampf um die konkurrierenden Lebensentwürfe des Dichters und des Ehemannes. Um nun nicht nur die Option des Dichters zu rechtfertigen, sondern, wie es für Kafkas Denkweise üblich ist, auch diese Rechtfertigung wiederum zu relativieren, weist er Felice darauf hin, dass der Gelehrte in der chinesischen Lyrik oft auch pejorativ als „Stubenhocker“ präsentiert wird (KAFKA 2003: 263). Das Gegenmodell ist der tapfere Kriegsheld, der zu seiner Ehefrau und zu seinen Kindern zurückkehrt. Die Bedeutung, welche die Einsamkeit des Schreibens für Kafka hat, gibt er Felice jedoch unmissverständlich zu verstehen:

Oft dachte ich schon daran, daß es die beste Lebensweise für mich wäre, mit Schreibzeug und einer Lampe im innersten Raume eines ausgedehnten, abgesperrten Kellers zu sein. Das Essen brächte man mir, stellte es immer weit von meinem Raum entfernt hinter der äußersten Tür des Kellers nieder. Der Weg um das Essen, im Schlafrock, durch alle Kellergewölbe hindurch wäre mein einziger Spaziergang. Dann kehrte ich zu meinem Tisch zurück, würde langsam und mit Bedacht essen und wieder gleich zu schreiben anfangen. (KAFKA 2003: 250)

Hier wird deutlich, dass die Einsamkeit des nächtlichen Lesens und Schreibens für Kafka, nicht für China, charakteristisch ist. Gleichwohl wird der Chinese in dem zitierten Gedicht für Kafka zum Emblem dieser Lebensweise. Kafkas China ist also zu großen Teilen das China dieses Gedichts, das den Konflikt zwischen einsamer geistiger Arbeit und geselligem bzw. intimen Beisammensein kennt und jenem den Vorzug gibt. Die Tugenden der Entsagung und Versenkung, der Einsamkeit und Stille, die den Taoismus kennzeichnen, macht Kafka sich hier zu eigen. Das Fremde wird nicht in seiner Umgebung, das chinesische Gedicht also nicht in seinem historischen und kulturellen Kontext interpretiert, sondern auf die eigene Situation angewendet. Diese Lesart hat den Vorzug, dass dadurch keine kulturellen Stereotype aufkommen können, 
denn Kafka rechtfertigt seine Dichterexistenz ja durch die Nachtarbeit des Chinesen in Jan-Tsen-Tsais Gedicht. ${ }^{3}$ Kafkas China ist hier also das China des einsamen Gelehrten. Aber es gibt noch mehr China bei Kafka.

\section{Beim Bau der chinesischen Mauer}

Borges schreibt: „Zwei Ideen - besser gesagt, zwei Obsessionen - beherrschen das Werk von Franz Kafka: erstens die Unterordnung, zweitens das Unendliche.“ (BORGES 1995:132) Unüberschaubare Hierarchien und unendliche Prozesse kennzeichnen Kafkas Texte insgesamt, man denke nur an die Unerreichbarkeit des Gesetzes oder des Schlosses, die auch mit undurchsichtigen Machtverhältnissen einhergeht. Vor allem das „Motiv der unendlichen Hintansetzung“ ist es, das nach Borges Kafkas Erzählungen beherrscht: so auch die kaiserliche Botschaft, die nie ankommt wegen der Personen, die den Boten behindern. In der Erzählung Beim Bau der chinesischen Mauer gebe es wiederholt Unendliches:

„Um den Marsch unendlich weit entfernter Heere aufzuhalten, befiehlt ein in der Zeit und im Raum unendlich entrückter Kaiser, daß unendliche Generationen unendlich lange eine unendliche Mauer errichten, die sein unendliches Reich umgeben soll.“ (BORGES 1995:133) Sind undurchsichtige Hierarchien und unendliche Aufschübe nun Motive, die Kafkas Bild von China kennzeichnen? Borges zufolge beherrschen sie sein Werk. Das heißt, sie sind wiederkehrende Motive, an denen Kafka, nicht China, zu erkennen ist. Um so mehr, als das Unendliche nicht nur motivisch eine Rolle spielt, sondern Borges zufolge auch für Kafkas Schreibpraxis gilt. Die Unabschließbarkeit der Romane wird damit zum poetologischen Programm erklärt.

Nun wäre jedoch zu fragen, ob gerade in dieser, Kafka eigenen Aufschub- und Unendlichkeitsfigur eine besondere Verbindung Kafkas zu China besteht: Worum geht es also in der 1917 entstandenen Erzählung Beim Bau der chinesischen Mauer und was ist daraus für Kafkas Sicht auf China abzuleiten? Die Erzählung gliedert sich in zwei Teile. Im ersten Teil ist von der chinesischen Mauer die Rede, die zum Schutz gegen die Nomaden aus dem Norden errichtet

3 Vgl. Rolf J. GoeBeL (1997:56): „Implicitly, then, Kafka’s preoccupation with the Chinese verses suggests that he seeks to break through the boundaries of the racial stereotypes and cultural provincialism of his time in order to explore the possible significance of a non-Western writer for justifying his own existential situation.“ 
wurde. ${ }^{4}$ Der Erzähler, der am Mauerbau beteiligt war, widmet sich der Frage nach der Zweckmäßigkeit des Teilbauprinzips, das darin besteht, an verschiedenen Enden mit dem Bau zu beginnen - mit der Folge, dass der Bau große Lücken aufweist. Im zweiten Teil geht es um das Kaisertum. Hier ist die Sage von der kaiserlichen Botschaft eingearbeitet, die Kafka in dem ErzählungenBand Der Landarzt 1919 gesondert publizierte. Kafkas Interesse an China wurde höchstwahrscheinlich durch den Reisebericht von Julius Dittmar aus dem Jahre 1910 sowie durch die von Hans Heilmann 1905 herausgegebene Sammlung chinesischer Lyrik geweckt (vgl. BINDER 1979:319 und 218). So ist dem Reisebericht beispielsweise zu entnehmen, dass die chinesische Mauer zum Schutz gegen „Überfälle der Nachbarn“ gebaut wurde, dass das Land schön und riesig ist und dass die Kaiserstadt aus mehreren schützenden Mauerrahmen besteht, während in der Gedichtanthologie etwa vom „staffelmäßig“ aufgebauten Schulsystem und einer „festgegründete[n] Beamtenhierarchie“ die Rede ist (vgl. MENG 1986:78).

All diese Motive nimmt Kafka auf, verwandelt aber die Größe des Landes in eine sagenhafte Unendlichkeit und die Mauerrahmen der Kaiserstadt in eine, mit Borges gesprochen, ,unendliche Entrücktheit’: „So groß ist unser Land, kein Märchen reicht an seine Größe, kaum der Himmel umspannt es. Und Peking ist nur ein Punkt, und das kaiserliche Schloß nur ein Pünktchen.“ (KAFKA 1994:74) Von dem Boten in der Sage heißt es,

immer noch zwängt er sich durch die Gemächer des inneren Palastes, niemals wird er sie überwinden und gelänge ihm das, nichts wäre gewonnen, die Treppen hinab müßte er sich kämpfen, und gelänge ihm das, nichts wäre gewonnen, die Höfe wären zu durchmessen, und nach den Höfen der zweite umschließende Palast, und wieder Treppen und Höfe und wieder ein Palast und soweiter durch Jahrtausende (KAFKA 1994:75f.).

Das Unökonomische des singulativen Erzählens, das nach dem anaphorischen Typus gestaltet ist und zudem im Irrealis steht, verdeutlicht die unendliche, vergebliche Anstrengung des Boten, zum Kaiser durchzudringen. Die räumliche Unerreichbarkeit wird durch die zeitliche Endlosigkeit, die das Wort „Jahrtausende“ evoziert, besiegelt.

Diese Stelle ist von entscheidender Bedeutung für die Frage nach Kafkas China-Bild. Denn einerseits ist hier nachzuweisen, wie Kafka seinen Erzähler

$4 \quad$ In Kafkas Text Ein altes Blatt, der ursprünglich mit Ein altes Blatt aus China überschrieben war, sind die Nomaden bereits „bis in die Hauptstadt gedrungen, die doch sehr weit von der Grenze entfernt ist“ (KAFKA 1994:81). Vgl. dazu AleXANDER HonOld (2005:217f.) und Gerhard OBERLin (2006:126f.). 
historisch korrekt von den geschachtelten Sicherheitszonen berichten lässt, andererseits übersteigert Kafka diese Schachtelung in eine Unendlichkeit des Scheiterns, die für viele seiner Erzählungen charakteristisch ist und von Borges als das spezifisch Kafkaeske angesehen wurde. Die Nähe der Sage von der kaiserlichen Botschaft zur Türhüterparabel liegt angesichts der Staffelung von Hindernissen und der Unmöglichkeit, das Ziel zu erreichen, auf der Hand.

Es ist also weniger Kafkas Sicht auf China, die sich in der Erzählung Beim Bau der chinesischen Mauer ausdrückt. Diese wäre nur an gängigen Topoi des damaligen Orientalismus-Diskurses festzumachen, bei denen die Größe, Fremdheit und das Herrschaftssystem Chinas dominieren (vgl. GoeBEL 1993:64). Vielmehr nimmt Kafka Merkmale Chinas zum Anlass, seine Denkfigur der unendlichen Hierarchie bzw. des unendlichen Aufschubs auszugestalten. China bietet sich dazu nicht nur aufgrund des Mauerbaus und des Kaisertums an, sondern auch, weil das Land für Europäer im frühen 20. Jahrhundert „eher ein fernes Gerücht als erfahrene Wirklichkeit“ (OBERLIN 2006:115) ist, und sich so als mythischer Inbegriff eines unverfügbar Fremden in besonderer Weise eignet.

Die meisten Interpreten der Erzählung sind darüber einig, dass China bei Kafka eine Chiffre für bestimmte politische Verhältnisse in Europa oder für das jüdische Volk (vgl. ALt 2005:580) oder, formaler und abstrakter gedacht, für Verstehens- und Schreib-Prozesse darstellt. Die Interpretationen laufen zumeist darauf hinaus, dass Kafkas Text nicht, oder zumindest nicht primär, eine literarische (Re-)Konstruktion des historischen China darstellt. Dem tschechischen Schriftsteller Jiří Gruša zufolge ist Kafkas China „nichts Exotisches“, sondern „Ausdruck Habsburger Verhältnisse, der ,konfuzianischen’ Institutionen des Kaiserreichs mit seinem Mandarinismus der Beamtenschaft" (GRUŠA 1983:10). ${ }^{5}$ Diese Position ist in der gegenwärtigen Kafka-Forschung weitgehend unbestritten. Die Analogie zwischen China und Österreich wird ferner dadurch begründet, dass die Endzeitstimmung der 1911 untergehenden Tsching-Dynastie, wie sie in Dittmars Reisebericht von 1912 vermittelt wird, jener der untergehenden österreichisch-ungarischen Doppelmonarchie wesenhaft ähnlich sei; denn mit dem Tod von Kaiser Franz Josef I. 1916 und der

Gruša hebt also vor allem das hierarchisch patriarchale Gesellschaftssystem mit dem riesenhaften Verwaltungsapparat als Gemeinsamkeiten hervor. Die gewaltige Größe der beiden Reiche und die Multiethnizität, welche zu Abgrenzungskämpfen und letztlich zum Zusammenbruch der Monarchien führt, sind weitere Gemeinsamkeiten. 
Niederlage im Ersten Weltkrieg 1918 ist die k.u.k. Donaumonarchie am Ende. Kafka reflektiert hier also möglicherweise die politische Situation in Europa mittels der China-Bilder, die er in der 1917 geschriebenen Erzählung entwirft (vgl. OBERLin 2006:116 sowie HoNOLD 2005:207f.). Interpretationen, die diese Ähnlichkeiten herausstellen, betonen eher die Verwandtschaft als die Dichotomie zwischen dem Westen und dem Osten.

Der Mauerbau wurde außerdem als Chiffre für Kafkas Schaffensprozess bedeutet, weil das Teilbauprinzip Ähnlichkeit mit dem nicht-linearen Schreibprozess und dem fragmentarischen Werk hat: z. B. im Falle des Fragment gebliebenen Romans Der Proceß hat Kafka mit dem ersten und letzten Kapitel begonnen und sodann, wiederum in Teilen, die Lücken gefüllt (vgl. STACH 2008:496).

Diese Interpretationslinie führt auf den ersten Blick von China weg, insofern hier die Inhalte beliebig erscheinen, während nur der Prozess des Bauens entscheidend ist. Aber Kafkas Nähe zu China besteht gerade in der Form, also in bestimmten Strukturen des Denkens und Schreibens. Welche Strukturen sind es nun aber, die sich in der Erzählung herauskristallisieren?

Die Erzählweise ist nicht sehr geradlinig und homogen. Der zu Beginn eingeführte geschichtswissenschaftliche Beschreibungsstil wird von zunehmend subjektiven Eindrücken, emotionalen Ausrufen und Spekulationen in einen Fluss des unabgeschlossenen Denkens gebracht. So versucht der Erzähler, die Gründe für das „System des Teilbaus“ zu finden und kommt zu dem vorläufigen Schluss, dass die „Führerschaft“ eigentlich in der Lage gewesen wäre, einen zusammenhängenden Mauerbau zu planen: „Bleibt also nur die Folgerung, daß die Führerschaft den Teilbau beabsichtigte.“ (KAFKA 1994:70f.) ${ }^{6}$ Sogleich wendet der Erzähler die Überlegung mit einen Einwand wieder um: „Aber der Teilbau war nur ein Notbehelf und unzweckmäßig.“ Nun steht der Erzähler vor einem Widerspruch, den er zugunsten der Führerschaft aufzulösen scheint, indem er einen Schluss zieht, der den Widerspruch in Wahrheit nicht löst: „Bleibt die Folgerung, daß die Führerschaft etwas Unzweckmäßiges wollte.“

Dass etwas nicht stimmt mit dieser Lösung, der zufolge Unzweckmäßiges bezweckt werde, merkt der Erzähler sogleich und kommentiert: „Sonderbare Folgerung, gewiß.“ Der Gedanke kommt hier nicht zum Stehen, sondern wird durch weitere Argumente fortgeführt, die ebenso wenig das Paradox aufheben, das sich hier abzeichnet. Der Grund dafür ist das vom Erzähler so benannte

6 Diese Angabe umfasst auch die unmittelbar anschließenden Zitate der Textpassage. 
„Endlose“ des Gebiets, „das hier zu durchlaufen wäre“, wenn für alles eine Erklärung gefunden werden müsste.

Zwei Denkstrukturen sind es also, die sich hier als wesentlich für diesen Text erweisen: das Unendliche des Denkweges, auf dem ein endgültiger Sinn immer weiter aufgeschoben wird, und das Paradoxe, das in der Bezweckung des Zwecklosen steckt und zugleich als Charakteristikum für die Chinesen bestimmt wird, wenn der Erzähler als Ergebnis seiner „vergleichenden Völkergeschichte“ feststellt: „daß wir Chinesen gewisse volkliche und staatliche Einrichtungen in einzigartiger Klarheit, andere wieder in einzigartiger Unklarheit besitzen“. Kafkas Texten ist ebenfalls eine einzigartige Klarheit zuzusprechen, insofern sie sprachlich einfach und deutlich sind, zugleich aber auch eine einzigartige Unklarheit, die darin besteht, vieldeutig und geheimnisvoll zu bleiben. Dies liegt an der Verwendung von Paradoxa, gleichnishafter Offenheit und zumeist dem Ausbleiben eines erklärenden Erzählerkommentars. In diesem Text drängt sich der Erzähler jedoch mit seiner Denkbewegung so in den Vordergrund, dass der Eindruck entstehen kann, der Text stelle seinen eigenen Erkenntnis- und Schreibprozess dar. ${ }^{7}$ Das Erzählte gerät schließlich in einen Sinn-Aufschub, wie Borges ihn im Begriff der unendlichen Hintansetzung als Kafka-typisch bestimmte.

\section{Dschuang Dsi: Gleichnisse, Paradoxien und bildkräftige Meta- phern}

Der britische Sinologe Arthur Waley sprach Elias Canetti zufolge von einem „,natürlichen’ Taoismus“ Kafkas (CANETTI 1983:76f.). Diese Ansicht wird zwar nicht weiter erörtert, aber im Vergleich mit taoistischen Texten von Laotse oder Dschuang Dsi zeigt sich tatsächlich eine stilistische Ähnlichkeit. Vor allem das Gleichnishafte, das Paradoxe und die ausdrucksstarken Metaphern sind hier hervorzuheben. Auf motivischer Ebene hat Canetti die Vorliebe für Tiere in den Geschichten bereits bemerkt. Sprachlich fällt vor allem die präzise Einfachheit auf, die mit gedanklicher Offenheit oder gar Rätselhaftigkeit einhergeht.

7 Vgl. Tillmann BuB (2006:416): „Der Text selbst muss als Mauer angesehen werden, deren Bau sich im Akt des Lesens immer wieder von Neuem unmittelbar vor den Augen des Lesers vollzieht.“ 


\section{Einer der berühmtesten Texte Dschuang Dsis ist Der Schmetterlingstraum:}

Einst träumte Dschuang Dschou, daß er ein Schmetterling sei, ein flatternder Schmetterling, der sich wohl und glücklich fühlte und nichts wußte von Dschuang Dschou. Plötzlich wachte er auf: da war er wieder wirklich und wahrhaftig Dschuang Dschou. Nun weiß ich nicht, ob Dschuang Dschou geträumt hat, daß er ein Schmetterling sei, oder ob der Schmetterling geträumt hat, daß er Dschuang Dschou sei, obwohl doch zwischen Dschuang Dschou und dem Schmetterling sicher ein Unterschied ist. So ist es mit der Wandlung der Dinge. (DschUANG DsI 1991:52 $)^{8}$

Hier wird eine erkenntnistheoretische Relativierung vorgeführt, die durch das wiederholte Überkreuzen von „Schmetterling“ und „Dschuang Dschou“ sprachlich erfahrbar gemacht wird. Das Gleichnishafte, Paradoxe und Bildkräftige zeigt sich in diesem Text besonders deutlich, da diese konkrete Situation durch den Nachsatz von der Wandlung der Dinge auf eine größere philosophische Tragweite hindeutet und der Widerspruch zwischen den beiden Varianten, denen zufolge einmal Dschuang Dschou und einmal der Schmetterling das Subjekt des Träumens sind, nicht aufgelöst wird.

Bei Kafka finden sich diese Formmerkmale und Denkfiguren auch häufig. Sie kennzeichnen geradezu seinen Stil. Ein Beispiel dafür, dass Kafkas Texte geläufige Vorstellungen auf den Kopf stellen, ist Die Wahrheit über Sancho Pansa (1917). Dieser Text verkehrt die Konstellation aus Cervantes’ Don Quijote ins Gegenteil:

Sancho Pansa, der sich übrigens dessen nie gerühmt hat, gelang es im Laufe der Jahre, durch Beistellung einer Menge Ritter- und Räuberromane in den Abendund Nachtstunden seinen Teufel, dem er später den Namen Don Quixote gab, derart von sich abzulenken, daß dieser dann haltlos die verrücktesten Taten aufführte, die aber mangels eines vorbestimmten Gegenstandes, der eben Sancho Pansa hätte sein sollen, niemandem schadeten. Sancho Pansa, ein freier Mann, folgte gleichmütig, vielleicht aus einem gewissen Verantwortlichkeitsgefühl, dem Don Quixote auf seinen Zügen und hatte davon eine große und nützliche Unterhaltung bis an sein Ende. (KAFKA 1994:167)

Ähnlich wie Dschuang Dsi das Subjekt-Objekt-Verhältnis zwischen Dschuang Dschou und dem Schmetterling verkehrt, dreht Kafka das Beziehungsverhältnis zwischen Don Quijote und seinem Begleiter um, indem er den einfachen

8 Die ursprüngliche Ausgabe ist von 1912 und war Kafka bekannt. Dschuang Dsi (ca. 365-290 v. Chr.) war ein chinesischer Philosoph und Dichter, Schüler des Laotse. Zusammen mit dessen Tao te king gilt das Buch vom südlichen Blütenland als Hauptwerk des Taoismus. ,Dschou‘ war sein Rufname. Vgl. hierzu die Einleitung von RichaRD WiLHELM (vgl. DSCHUANG Dsi 1991). 
Bauern zum lenkenden Subjekt des Geschehens macht. Bei Cervantes ist Sancho Pansa der Knecht, in Kafkas Text wird er zum listigen Drahtzieher, während Don Quijote zu seinem vom ihm gebändigten Teufel wird. Die Frage, bei wem die Souveränität der Erkenntnis liegt, wird also in beiden Texten durch eine paradoxe, d.h. den Erwartungen zuwiderlaufende Umkehrungsfiguration aufgeworfen.

In der Form bestehen also Ähnlichkeiten zwischen Dschuang Dsis und Kafkas Texten, die als repräsentativ gelten können. Dies sind vor allem die paradoxe Relativierung der Perspektiven und des Geläufigen. Der Unterschied zwischen den Texten besteht allerdings darin, dass bei Dschuang Dsi zumeist eine deutliche moralische Richtung eingeschlagen wird. Im Schmetterlingstraum manifestiert sich diese in dem Nachsatz über die Wandlung der Dinge. Bei Kafka gibt es solche Nachsätze nicht, und wenn es sie gibt, dienen sie eher dazu, sich gegenseitig zu kippen und zu verunklären, als dazu, eine Weisheit zu festigen. ${ }^{9}$

\section{Fazit}

Obwohl Kafka sich mit China im Hinblick auf gesellschaftliche Gefüge und Denkweisen auseinandersetzt und dies zu Beginn des 20. Jahrhunderts tut, in einer Zeit also, in der China aufgrund seiner politischen Umbruchsituation international in den Blick gerät, steht sein Bild von China im Zeichen einer Projektion seines eigenen dichterischen Selbstbildes. Das Gedicht Jan-TsenTsais, In tiefer Nacht, bietet für Kafka eine Projektionsfläche aufgrund der Nachtarbeit, durch die er Felice gegenüber seine Dichterexistenz zu rechtfertigen sucht. Die Einsamkeit des Schreibens verlangt dem bürgerlichen Eheleben gegenüber eine Rechtfertigung, und Kafka findet sie bei dem chinesischen Dichter. Darin sieht er einerseits keine Eigentümlichkeit des Chinesischen, sondern eher eine universale Notwendigkeit dichterischen Schaffens, andererseits wird Jan-Tsen-Tsai durch das Gedicht dergestalt zu einem Gewährsmann für Kafka, dass er sich selbst als einen Chinesen bezeichnet. Auch die Analyse der Erzählung Beim Bau der chinesischen Mauer hat ergeben, dass Kafka vom damaligen China-Diskurs nur einige Merkmale aufgreift wie die unüberschaubare Größe, die Hierarchien und unverfügbare Fremdheit, um seine eigene

9 Dieser Text war ein Beitrag für die Tagung „Das Bild der Anderen. Die wechselseitige kulturelle Wahrnehmung Chinas und Deutschlands“, die vom 29. September bis 2. Oktober 2010 in Königswinter unter der Leitung von Prof. Dr. Georg Braungart stattfand. 
Denk- und Schreibweise des unendlichen Aufschubs auszubuchstabieren. Das Teilbauprinzip des Mauerbaus kann aufgrund seiner Strukturähnlichkeit mit dem nicht-linearen, fragmentarischen Schreibprozess Kafkas als Metapher für dessen Dichtung gedeutet werden. Abschließend findet diese These Bestätigung darin, dass Kafkas Texte insgesamt Ähnlichkeit mit Denkstrukturen des Taoismus haben, wie sie beispielsweise im Werk von Dschuang Dsi zum Ausdruck kommen. Feststehende Vorstellungen, beispielsweise davon, bei wem die Souveränität der Erkenntnis liegt, wer also das Objekt und das Subjekt des Denkens ist, werden bei Kafka wie bei Dschuang Dsi auf den Kopf gestellt. Dies ist nicht nur eine gedankliche Parallele, sondern eine Ähnlichkeit im Umgang mit der ästhetischen Form der Paradoxa. Kafkas Bild von China wird also in allen angeführten Beispielen heimgeholt in Kafkas Bild von Kafka.

\section{Literatur}

Alt, Peter-André (2005): Franz Kafka. Der ewige Sohn. Eine Biographie. München. Binder, HARTMUT (1979) (ed.): Kafka-Handbuch. 2 Bde. Bd. 1: Der Mensch und seine Zeit. Stuttgart.

Borges, JoRge LuIs (1992): Inquisitionen. Übersetzt von Karl August Horst und Gisbert Haefs. Frankfurt am Main.

Borges, Jorge LuIs (1995): Persönliche Bibliothek. Übersetzt von Gisbert Haefs. Frankfurt am Main.

Bub, Tillmann (2006): Eine Mauer des Verstehens. Die literarische Gestaltung eines hermeneutischen Grundproblems in Franz Kafkas „Beim Bau der chinesischen Mauer“. In: Wirkendes Wort 56:403-420.

CANetTi, Elias (1983): Der andere Prozeß. Kafkas Briefe an Felice. Leipzig.

DschuANG Dsi (1991): Das wahre Buch vom südlichen Blütenland. Übersetzt und erläutert von Richard Wilhelm. München.

Goebel, Rolf J. (1993): Constructing Chinese History: Kafka's and Dittmar's Orientalist Discourse. In: PMLA, Vol. 108/1:59-71.

GRUŠA, JIŘí (1983): Franz Kafka aus Prag. Frankfurt am Main.

Honold, Alexander (2005): Kafkas vergleichende Völkerkunde: „Beim Bau der chinesischen Mauer“. In: DunKer, Axel (ed.): (Post-)Kolonialismus und Deutsche Literatur. Impulse der angloamerikanischen Literatur- und Kulturtheorie. Bielefeld, 202-218.

KAFKA, FrANZ (1994): Beim Bau der chinesischen Mauer. Gesammelte Werke in zwölf Bänden. Nach der kritischen Ausgabe herausgegeben von HANS-GERD KOCH. Frankfurt am Main. Bd. 6: Beim Bau der chinesischen Mauer und andere Schriften aus dem Nachlaß. 
KaFKa, Franz (1994): Die Wahrheit über Sancho Pansa. Gesammelte Werke in zwölf Bänden. Nach der kritischen Ausgabe herausgegeben von HANS-GERD KOCH. Frankfurt am Main. Bd. 6: Beim Bau der chinesischen Mauer und andere Schriften aus dem Nachlaß.

KAFKA, FrANZ (1994): Ein altes Blatt. Gesammelte Werke in zwölf Bänden. Nach der kritischen Ausgabe herausgegeben von HANs-GERD KocH. Frankfurt am Main. Bd. 6: Beim Bau der chinesischen Mauer und andere Schriften aus dem Nachlaß.

KAFKA, FRANZ (2003): Briefe an Felice und andere Korrespondenz aus der Verlobungszeit. Herausgegeben von ERICH HELLER und JÜRGEN BORN. Frankfurt am Main.

Meng, WeiYAn (1986): Kafka und China. München.

OBerLin, Gerhard (2006): Die Grenzen der Zivilisation. Franz Kafkas Erzählung „Beim Bau der chinesischen Mauer“ und „Ein altes Blatt“. In: Orbis Litterarum, Band 61, 2:114-132.

StACH, ReINER (2008): Kafka. Die Jahre der Erkenntnis. Frankfurt am Main. 